\title{
Subjective Sense of Memory Strength and the Objective Amount of Information Accurately Remembered Are Related to Distinct Neural Correlates at Encoding
}

\author{
Shaozheng Qin, ${ }^{1,2,3}$ Hein J. F. van Marle, ${ }^{1,2,4}$ Erno J. Hermans, ${ }^{1,2,5}$ and Guillén Fernández ${ }^{1,2}$ \\ ${ }^{1}$ Donders Institute for Brain, Cognition and Behaviour, Radboud University Nijmegen, and ${ }^{2}$ Department of Cognitive Neuroscience, Radboud University \\ Nijmegen Medical Centre, 6500 HB Nijmegen, the Netherlands, ${ }^{3}$ Department of Psychiatry and Behavioral Sciences, Stanford University School of Medicine, \\ Stanford, California 94303, ${ }^{4}$ Department of Psychiatry, Academic Medical Center, University of Amsterdam, 1105 AZ Amsterdam, the Netherlands, and \\ ${ }^{5}$ Department of Psychology, New York University, New York, New York 10003
}

Although commonly used, the term memory strength is not well defined in humans. Besides durability, it has been conceptualized by retrieval characteristics, such as subjective confidence associated with retrieval, or objectively, by the amount of information accurately retrieved. Behaviorally, these measures are not necessarily correlated, indicating that distinct neural processes may underlie them. Thus, we aimed at disentangling neural activity at encoding associated with either a subsequent subjective sense of memory strength or with a subsequent objective amount of information remembered. Using functional magnetic resonance imaging (fMRI), participants were scanned while incidentally encoding a series of photographs of complex scenes. The next day, they underwent two memory tests, quantifying memory strength either subjectively (confidence on remembering the gist of a scene) or objectively (the number of details accurately remembered within a scene). Correlations between these measurements were mutually partialed out in subsequent memory analyses of fMRI data. Results revealed that activation in left ventral-lateral prefrontal cortex and temporoparietal junction predicted subsequent confidence ratings. In contrast, parahippocampal and hippocampal activity predicted the number of details remembered. Our findings suggest that memory strength may reflect a functionally heterogeneous set of (at least two) phenomena. One phenomenon appears related to prefrontal and temporoparietal top-down modulations, resulting in the subjective sense of memory strength that is potentially based on gist memory. The other phenomenon is likely related to medial-temporal binding processes, determining the amount of information accurately encoded into memory. Thus, our study dissociated two distinct phenomena that are usually described as memory strength.

\section{Introduction}

Strong memories are often characterized as those memories that remain stable, while time or interference eliminates weak memories. Beside this durability concept, memory strength has also been conceptualized by retrieval characteristics (Ebbinghaus, 1885; Schacter, 2001). For instance, a strong memory can be defined as a memory that is retrieved with high subjective confidence, or objectively, with a high amount of information accurately remembered. Behaviorally, however, such subjective and objective measures are not necessarily correlated with each other (Tulving, 1981; Schacter, 1995; Kensinger, 2008), suggest-

\section{Received May 20, 2010; revised April 25, 2011; accepted April 28, 2011.}

Author contributions: S.Q., E.J.H., and G.F. designed research; S.Q. and H.J.F.v.M. performed research; S.Q. and H.J.F.v.M. contributed unpublished reagents/analytic tools; S.Q. and E.J.H. analyzed data; S.Q., E.J.H., and G.F. wrote the paper.

This work was supported by Grants provided by the Netherlands Organisation for Scientific Research (NWO): No. 918.66.613,451.07.019, and 446.10.010. We thank Dr. Jianhui Wu for his helpful discussions, and three anonymous referees for their thoughtful suggestions and comments.

Correspondence should be addressed to Dr. Shaozheng Qin, Department of Psychiatry and Behavioral Sciences, Stanford University School of Medicine, Stanford, CA 94303. E-mail: szqin@stanford.edu.

DOI:10.1523/JNEUROSCI.2587-10.2011

Copyright $\odot 2011$ the authors $\quad 0270-6474 / 11 / 318920-08 \$ 15.00 / 0$ ing that they may rely on distinct neural processes and partly different representations.

Several well documented phenomena of memory distortions indicate that the dissociation between these subjective and objective measures is relevant. High confidence of introspective experience, for instance, is not always veridical, as in cases of eyewitness misidentification or illusory memory (Loftus, 1979; Schacter, 1995). One explanation for this is that subjective confidence may rely on processes related to remembering the gist of an episode, whereas objectivity of memories is based on memory formation for specific details. Converging evidence from neuropsychological and functional neuroimaging studies on memory distortions suggest distinct neural correlates associated with false and true memories. Particularly, studies on gist-based false recognition memory suggest that a frontal-parietal network appears related to the subjective sense of remembrance in absence of veridicality (Schacter et al., 1996; Cabeza et al., 2001; Kim and Cabeza, 2007; Schacter and Addis, 2007). In contrast, activation in the medial temporal lobe (MTL), specifically the parahippocampal cortex, is associated with the successful encoding and retrieval of true memories (Cabeza et al., 2001; Kao et al., 2005; Eichenbaum et al., 2007; Squire et al., 2007), indicating an asso- 
ciation with objective veridicality of memories. These studies probing neural correlates of false and true memory, however, do not address the question of whether neural operations at encoding distinguish between subsequent memories characterized either by subjective sense of remembrance or objective amount of information remembered.

We therefore developed a paradigm that allowed us to dissociate memory encoding-related neural activity associated with memory strength based on either a subjective or an objective measure. During event-related fMRI, participants learned incidentally a series of photographs of complex scenes. Two different memory tests were completed $1 \mathrm{~d}$ later. In one test, participants rated the subjective confidence with which they remembered having seen photographs corresponding with one-sentence written gist descriptions. In a second test, participants were required to distinguish three true from three false details of each scene. Importantly, because these two measures are likely correlated, we orthogonalized both measures with respect to each other before entering them as trial-based parametric modulations in subsequent memory analyses of fMRI data. This allowed us to identify and dissociate neural activity associated with either subjective or objective measures of memory strength. Drawing on previous findings of distinct neural signatures of false and true memories, we expected that prefrontal activity would predict memory strength based on subjective measures (confidence of remembering the gist), and that MTL activity would predict memory strength based on objective measures (number of details accurately remembered).

\section{Materials and Methods}

Participants. Twenty-two young, healthy, male volunteers (aged 19-36 years; mean \pm SD, $22.7 \pm 4.01$ years) with normal (or corrected) vision participated in this study. Participants were healthy and reported no history of neurological or psychiatric disease. Participants gave written informed consent in accordance with the requirements of the local institutional review board (CMO Arnhem-Nijmegen) and the declaration of Helsinki. Data of two participants were excluded from further analysis: one due to poor memory performance (hit rate at chance level of 0.5 ) and the other one due to excessive head movement during scanning $(>4 \mathrm{~mm}$ within one run).

Stimuli. Initially, we carefully selected 200 color photographs of scenes depicting distinct meaningful activities related to either humans or animals from a commercially available image database (http://www. shutterstock.com/). For each picture, we created a sentence of 5-10 words describing the central meaning ("gist") of the scene on the basis of the following two criteria (Adolphs et al., 2001, 2005): first, the sentence should be sufficient to identify the photograph and to distinguish it from the other scenes. Second, the sentence should not include any peripheral detail. Another identically structured set of sentences (based on different photographs that were not used in this study) was created as lures for the memory test. Moreover, for the memory test on details, eight details were identified in each picture. For each detail, we made both a true and an adapted false (but equally plausible) detail, resulting in eight pairs of true and false details for each picture. These eight pairs per picture were obtained from a separate pilot study, in which 10 additional participants rated the likelihood that these true and false details would occur in a scene described by the one-sentence description, but without having seen the actual photographs of the scenes. The difference of likelihood ratings between true and false details was calculated for each pair and across participants. Only true and false details whose likelihood ratings did not differ significantly $(p>0.05)$ were used for the actual experiment (see Table 1 for an example). Written gist descriptions of pictures that were rated as unclear descriptions by any participant were furthermore excluded. The final stimulus set used for the fMRI experiment consisted of 150 scenes (plus 10 extra scenes for the training set) with one-sentence
Table 1. An example of written descriptions for the gist and associated true and fictitious details of a complex scene

\begin{tabular}{llll}
\hline \multirow{4}{*}{ Gist } & Pairs of details & & \\
\cline { 2 - 3 } & True & Figctitious & Sig. \\
\hline A man nearby his glider & Man wears black T-shirt & Man wears white T-shirt & 0.12 \\
& Man wears white hat & Man wears black hat & 0.18 \\
& Man with red backpack & Man with blue backpack & 0.23 \\
& Trees at the background & Houses at the background & 0.13 \\
& Cloudy sky & Blue sky & 0.50 \\
& Cabin of glider open & Cabin of glider closed & 0.11 \\
\hline
\end{tabular}

Sig. stands for the significance of the difference of the likelihood ratings between actual and fictitious details, which was obtained from a separate pilot study.

gist descriptions and six pairs of true and false details. Luminance of all selected pictures was equalized.

Procedure and tasks. The experiment consisted of a study phase on the first day (day 1) and two memory tests on the consecutive day (day 2) (Fig. 1). During the study phase, participants were scanned while performing an incidental encoding task on 150 sequentially and centrally presented photographs (presentation time: $6 \mathrm{~s}$, mean intertrial interval, $6 \mathrm{~s}$, randomly varying between 3 and $9 \mathrm{~s}$ in steps of $1 \mathrm{~s}$ ). Participants were instructed to imagine themselves being in the scene as vividly as possible, and to make a judgment of how much they would like to be present in that scene on a four-point scale (i.e., 1 , would like it very much; 2 , would like it; 3, would not like it; 4 , would not like it at all). To ensure that participants did not expect any subsequent memory tests, they were told that they participated in a study on mental imagery. Moreover, they were informed that their eye movements would be recorded using an eye tracker. The study phase was divided into three runs of 50 trials each. Within each run, 10 null events of $6 \mathrm{~s}$ duration were pseudorandomly intermixed to minimize potential effects of expectation and optimize contrast-to-noise ratio of event-related fMRI signal. Each run lasted $\sim 12$ min. To familiarize participants beforehand with the procedure, they were trained twice using 10 trials, which were not used in the actual experiment.

Approximately at the same time on the consecutive day (mean interval: $23.5 \pm 1.2 \mathrm{~h}$ ), participants came back and performed two surprise memory tests. Before the start of these tests, participants were asked whether they had expected a subsequent memory test while being scanned the day before. None of them had expected any memory test(s). Thereafter, the experimenter debriefed the participants by explaining the rationale of the memory tests.

The first test was included to assess subjective confidence on remembering the gist of a studied scene (i.e., subjective measure of memory strength). In this task, 150 written gist descriptions of studied scenes were randomly intermixed with 150 descriptions of scenes that were not studied before. Participants were asked to judge whether each description was associated with a scene studied before or not (i.e., "Yes" or "No") and give a confidence rating on a visual analog scale ranging from $0 \%$ to $100 \%$ by moving the cursor via a mouse movement to the appropriate position (Fig. 1). This task was self-paced with a trial duration limited to a range between 2 and $8 \mathrm{~s}$.

The second memory test was included to assess how much detail the participants remembered for each scene. Here, the written descriptions of each studied scene were presented sequentially at the center of the screen for $1 \mathrm{~s}$. After each description, three true and three false details related to the same scene were presented randomly intermixed one after another in the center of the screen. Participants were instructed to judge whether each detail was either true or false (Fig. 1). The selection of three true and three false details was randomized separately for each scene and participant. To reduce the expectancy that $50 \%$ of details were correct, we randomly intermixed descriptions of practice trials, for which either all six details were true, or all six details were false. The detail memory test was divided into three blocks of 50 trials each. The task was self-paced limited to a presentation time per detail between 2 and $8 \mathrm{~s}$.

Memory performance data analysis. For the memory test for identification of one-sentence written gist descriptions, numbers of hits, misses, false alarms, and correct rejections and hit rate and false alarm rate across 
all trials are shown in Table 2. Two different methods were used to derive a performance measure: hit rate minus false alarm rate (referred to as accuracy unless specified otherwise) and hit rate divided by hit rate plus false alarm rate (referred to as proportion correct) (Wixted and Ebbesen, 1991; Wixted et al., 2010). Note that chance level for accuracy is zero (range -1 to 1 ), whereas chance level for proportion correct is 0.5 (range $0-1$ ). Confidence ratings $(1-100 \%)$ of each participant were grouped into four bins with increasing ratings to accommodate variability of their distribution. These four bins corresponded with equal lengths on the visual analog scale and thus reflected each participant's individual distribution of confidence ratings. Performance measures within each of these four different confidence levels of remembering the gist are also summarized in Table 2, in which hit rate and false alarm rate were based on the denominator with hits plus misses or false alarms plus correct rejections within each of four different confidence bins. The trial-based confidence ratings (for hit trials) were used in fMRI data analyses described below.

We subsequently used the detail memory test to derive an objective measure of memory strength. Performance measures for this test were calculated on a trial-by-trial basis (i.e., for each true gist description) across the six (three true, three false) detail items that were probed. Table 2 summarizes these averaged performance data across all participants and across all trials. Additionally, trialbased detail memory performance measures are presented separately for the four subjective confidence levels resulting from four levels of confidence of remembering the gist described above. Again, we used two different scoring methods to calculate trial-based accuracy and proportion correct. The resulting trial-based accuracy and proportion correct scores were also used in fMRI data analyses.

Memory performance in the two memory tests was submitted separately for statistical testing to SPSS 16.0 (SPSS). We calculated one-sample $t$ tests against chance levels for hit rate and both accuracy measures described above. In addition, correlations between confidence ratings of remembering the gist and objective detail memory performance were initially calculated on a trial-by-trial basis for each participant. Individual correlation coefficients were then Fisher's $z$-transformed for further statistical testing.

fMRI data acquisition. Whole-brain $\mathrm{T} 2{ }^{*}$-weighted gradient echo EPI images with blood oxygenation level-dependent contrast were acquired with a Siemens Trio 3.0 T MR scanner using an ascending slice acquisition sequence ( 37 axial slices, TR: $2.18 \mathrm{~s}$; TE: $25 \mathrm{~ms}$; flip angle: $90^{\circ}$; slice matrix size: $64 \times 64$; slice thickness: $3.0 \mathrm{~mm}$; slice gap: $0.3 \mathrm{~mm}$; FOV: $212 \times 212 \mathrm{~mm})$. The study phase was divided into three runs of 336 volumes each. High-resolution structural images $(1 \times 1 \times 1 \mathrm{~mm})$ were acquired using a T1-weighted three-dimensional magnetization-prepared rapid gradient-echo sequence (TR: $2.3 \mathrm{~s}$; TE: $2.96 \mathrm{~ms}$, flip angle: $8^{\circ}$, FOV: $256 \times 256 \times 192 \mathrm{~mm})$.

fMRI data analysis. Image preprocessing and statistical analysis was performed using SPM5 (http://www.fil.ion.ucl.ac.uk/spm). The first five EPI volumes were discarded to allow for T1 equilibration. Remaining functional images were rigid-body motion corrected and the mean image was coregistered to each participant's T1-weighted MR image. Subsequently, images were corrected for slice acquisition timing, transformed into a common stereotactic space (using the MNI152 T1-template), and resampled into $2 \mathrm{~mm}$ isotropic voxels. Finally, images were spatially smoothed by convolving with an isotropic 3D Gaussian kernel ( $8 \mathrm{~mm}$ full width at half maximum). The data were statistically analyzed using general linear models (GLMs) and statistical parametric mapping (Friston et al., 1995).
Day 1

Day 2

Memory test for probing confidence

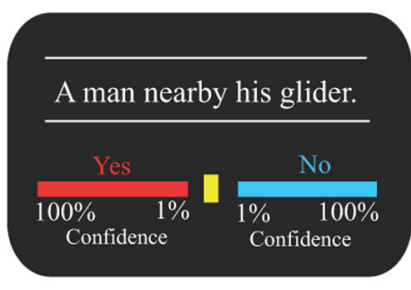

Memory test for probing detail memory

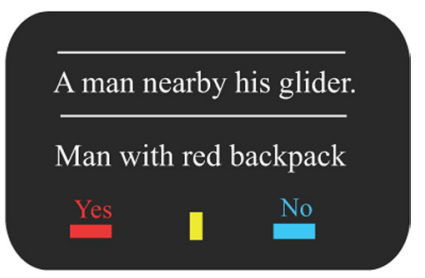

$3.0-9.0 \mathrm{~s}$ the first day (day 1) and two subsequent memory tests on the consecutive day (day 2). In the study phase, participants were scanned while they incidentally encoded 150 complex memory test for written gist descriptions of scenes with subjective confidence ratings on a visual analog scale (i.e., 1-100\%) randomly intermixed with lures. Next, they were given a second memory test assessing how many details were accurately remembered for each scene (see Materials and Methods for more details).

To tackle the question about differential neural correlates of memory formation associated with either subsequent subjective or objective measure, we first conducted an event-related parametric analysis with linearly orthogonalized subjective (confidence ratings in memory test for identification of gist descriptions) and objective (trial-based detail memory accuracy) measures as parametric modulations separately. Linear orthogonalization was calculated as follows. First, the linear regression between detail memory accuracy $\left(X_{1}\right)$ and subjective confidence of remembering the gist $\left(X_{2}\right)$ was expressed as $X_{2}=a \times X_{1}+b$. The orthogonalized accuracy score $\left(X_{1 \mathrm{o}}\right)$ was subsequently calculated as $X_{1 \mathrm{o}}=X_{1}-$ $\left(a \times X_{1}+b\right)$ and vice versa. Based on the memory test for one-sentence written gist descriptions of studied scenes, encoding trials were first sorted into two categories (written gist description remembered and forgotten). Then, the two conditions were modeled as separate regressors and convolved with the canonical hemodynamic response function in SPM5. Trial-based confidence scores reflecting subjective sense of memory strength were first standardized and then linearly transformed to partial out their shared covariance (see behavioral results for correlation coefficient) with accuracy scores resulting from the objective detail memory test. Subsequently, these orthogonalized confidence scores were included into a first-level GLM as linear parametric modulation covariates (for the remembered items regressor only) to account for neural activity specifically related to the subjective sense of memory strength. Similarly, trial-based detail memory accuracy scores were standardized and orthogonalized with respect to the confidence ratings, and then included as parametric modulations in a separate first-level GLM to account for neural activity uniquely associated with the objective measure of amount of information remembered. Additionally, realignment parameters were included in all models to account for movementrelated variability. Furthermore, we used high-pass filtering with a cutoff of $1 / 128 \mathrm{~Hz}$, global intensity normalization, and serial correlations correction using a first-order autoregressive (AR[1]) model.

Contrast parameter images for scene encoding (vs fixation baseline), successful memory formation (one-sentence gist description remembered vs forgotten), and parametric modulations corresponding with subjective and objective measures were generated from within the first- 
Table 2. Average performance (mean \pm SEM) for the memory test for identifying gist descriptions, and corresponding trial-based and grand accuracy in the detail memory test, across all trials and within four confidence levels of remembering the gist $(N=20)$

\begin{tabular}{|c|c|c|c|c|c|}
\hline & \multirow[b]{2}{*}{ Across all trials } & \multicolumn{4}{|c|}{ Separated for four levels of confidence of remembering the gist } \\
\hline & & Unsure & Somewhat sure & Sure & Very sure \\
\hline \multicolumn{6}{|c|}{ Memory test of identifying the gist } \\
\hline Hits & $98.95 \pm 3.01$ & $14.70 \pm 1.91$ & $14.10 \pm 0.81$ & $10.70 \pm 1.14$ & $59.45 \pm 4.22$ \\
\hline Misses & $51.05 \pm 3.01$ & $13.30 \pm 2.21$ & $12.05 \pm 1.38$ & $10.4 \pm 1.66$ & $15.30 \pm 2.38$ \\
\hline $\mathrm{FA}$ & $29.65 \pm 3.87$ & $12.80 \pm 1.79$ & $7.80 \pm 1.11$ & $4.70 \pm 1.02$ & $5.35 \pm 1.50$ \\
\hline$C R$ & $120.35 \pm 3.87$ & $19.25 \pm 4.38$ & $24.45 \pm 3.35$ & $21.95 \pm 3.06$ & $53.25 \pm 7.65$ \\
\hline$H R$ & $0.670 \pm 0.020$ & $0.555 \pm 0.031$ & $0.563 \pm 0.032$ & $0.541 \pm 0.044$ & $0.803 \pm 0.027$ \\
\hline FAR & $0.197 \pm 0.026$ & $0.461 \pm 0.539$ & $0.250 \pm 0.039$ & $0.187 \pm 0.037$ & $0.164 \pm 0.054$ \\
\hline Accuracy & $0.473 \pm 0.018$ & $0.086 \pm 0.049$ & $0.308 \pm 0.039$ & $0.328 \pm 0.043$ & $0.599 \pm 0.048$ \\
\hline$P C$ & $0.785 \pm 0.019$ & $0.576 \pm 0.031$ & $0.716 \pm 0.031$ & $0.764 \pm 0.033$ & $0.869 \pm 0.029$ \\
\hline \multicolumn{6}{|c|}{ Trial-based detail memory } \\
\hline$H R$ & $0.612 \pm 0.015$ & $0.489 \pm 0.031$ & $0.592 \pm 0.029$ & $0.531 \pm 0.035$ & $0.625 \pm 0.018$ \\
\hline FAR & $0.434 \pm 0.013$ & $0.434 \pm 0.025$ & $0.477 \pm 0.025$ & $0.463 \pm 0.031$ & $0.407 \pm 0.017$ \\
\hline Accuracy & $0.177 \pm 0.015$ & $0.055 \pm 0.021$ & $0.115 \pm 0.025$ & $0.068 \pm 0.027$ & $0.229 \pm 0.015$ \\
\hline$P C$ & $0.596 \pm 0.007$ & $0.522 \pm 0.013$ & $0.563 \pm 0.016$ & $0.514 \pm 0.027$ & $0.615 \pm 0.011$ \\
\hline
\end{tabular}

$N$, Number of participants; FA, false alarm; CR, correct rejection; HR, hit rate; FAR, false alarm rate; accuracy, calculated by HR - FAR; PC (proportion correct), calculated by HR divided by HR plus FAR.

Table 3. Number of encoding trials for each of the seven levels of trial-based detail memory accuracy $(N=20)$

\begin{tabular}{llllllll}
\hline & -1.0 & $-2 / 3$ & $-1 / 3$ & 0 & $1 / 3$ & $2 / 3$ & 1.0 \\
\hline Mean (SEM) & $0.60(0.19)$ & $4.65(0.55)$ & $16.10(0.75)$ & $27.80(1.35)$ & $28.15(1.32)$ & $17.70(1.15)$ & $3.95(0.63)$ \\
Simulated mean (SEM) & $1.55(0.047)$ & $9.28(0.28)$ & $23.19(0.71)$ & $30.92(0.94)$ & $23.19(0.71)$ & $9.28(0.28)$ & $1.55(0.047)$ \\
\hline
\end{tabular}

These data are restricted to trials on which the one-sentence written gist description was correctly recognized. Trial-based detail memory accuracy was calculated by hit rate minus false alarm rate. Simulated means (and SEM) represent the distribution under the null hypothesis of no detail memory. N, Number of participants.

level GLMs. Subsequently, those parameter images were submitted to the second-level group analyses using one-sample $t$ tests that treated participants as a random variable. In the whole-brain analysis, results from these analyses were initially thresholded at $p<0.001$ (uncorrected), and then cluster sizes were used for statistical inferences. Unless otherwise specified, only clusters significant at $p<0.05$ (corrected for multiple non-independent comparisons) (Worsley et al., 1996) are reported with the Montreal Neurological Institute (MNI) coordinates of their local maxima. Given our a priori hypothesis regarding the MTL (hippocampus and parahippocampal cortex) and the ventral lateral prefrontal cortex (PFC), three separate masks were used to define search regions for small volume corrections. Bilateral hippocampus and bilateral parahippocampal cortex were both anatomically defined as detailed by Qin et al. (2009). Because functional subregions within the PFC are less clearly defined anatomically, we created a region of interest (ROI) consisting of all voxels within an anatomical mask of the ventral lateral PFC as defined in the Automatic Anatomical Labeling template (Tzourio-Mazoyer et al., 2002) that activated at the group level in the contrast of all encoding trials versus low-level baseline. Note that this contrast is orthogonal to the subjective and objective measures of interest.

Additionally, we performed a complementary analysis using a categorical model that allowed us to confirm and better illustrate activation patterns in the PFC and the MTL related to subjective and objective measures, because such patterns cannot be derived from orthogonalized event-related parametric analyses in a straightforward manner. For this model, we split each participant's trials into four categories based on high versus low scores on the two relevant dimensions (subjective and objective measures). For the confidence ratings of gist description identification, we regrouped the four confidence level bins into two categories of "lower" ("unsure," "somewhat sure," or "sure") versus "higher" ("very sure") confidence (Table 2). Similarly, the trial-based detail memory accuracy scores (based on hits minus false alarms) were grouped into two categories of "lower" ( -1 to 0$)$ and "higher" (0.33-1) levels (Table 3).

In this first-level categorical GLM, four separate regressors of interest were included corresponding with lower and higher levels of the subjective (i.e., confidence on remembering the gist) and the objective (i.e., detail memory accuracy) measure only for trials that were later remembered: (1) higher scores for both subjective and objective measures (highhigh); (2) higher scores for the subjective measure but lower scores for the objective measure (high-low); (3) lower scores for the subjective measure but higher scores for the objective measure (low-high); (4) lower scores for both subjective and objective measures (low-low). Remaining trials were modeled as a condition of no interest. Settings for this GLM analysis were otherwise identical to the parametric GLMs described above. Parameter estimates associated with each of the four regressors of interest were extracted from the three aforementioned ROIs (i.e., the bilateral hippocampus, the bilateral parahippocampal cortex, and the left ventral lateral PFC) at the individual level using MarsBar (http://marsbar.sourceforge.net) (Brett et al., 2002) and averaged across voxels within regions. Subsequently, extracted data were visualized in bar graphs and submitted to separate 2 (subjective: low vs high) by 2 (objective: low vs high) repeated-measures ANOVAs for further statistical testing in SPSS.

All parametric fMRI data analyses were performed using trial-based measures of both accuracy and proportion correct (see above for details). However, the results were very similar and all data presented in tables and figures were therefore based on only one of the two methods (accuracy, i.e., hit rate minus false alarm rate). For the categorical model, both performance measures yield the same results because the grouping of trials based on objective detail memory turns out identical.

\section{Results}

\section{Memory performance}

Descriptive statistics of performance data for the two different memory tests are listed in Table 2 . In addition to averages (and SEM) across all trials, all performance measures were also calculated separately within the four separate confidence bins of remembering the gist. One-sample $t$ tests showed that overall accuracy and proportion correct for identifying gist descriptions across all trials was well above chance level (both $t_{(19)}>14.67$, both $p<0.001)$. As can be seen in Table 2 , accuracy and proportion correct for identifying gist descriptions improved with increasing levels of confidence.

Overall performance in the detail memory test for each participant was calculated as the mean of all trial-based detail memory accuracy and proportion correct scores (Table 2). When tested across participants, accuracy and proportion correct on this test were also well above chance level (both $t_{(19)}>4.99$, both 
Table 4. Brain activations related to encoding of complex pictures (all trials during encoding vs fixation)

\begin{tabular}{|c|c|c|c|c|c|c|}
\hline \multirow[b]{2}{*}{ Brain regions } & \multirow[b]{2}{*}{$\mathrm{R} / \mathrm{L}$} & \multirow[b]{2}{*}{ BA } & \multirow[b]{2}{*}{$t$ values } & \multicolumn{3}{|c|}{ MNI152 coordinates } \\
\hline & & & & $x$ & $y$ & $z$ \\
\hline \multirow{3}{*}{ Occipital cortex } & L & 19 & $18.12^{* * *}$ & -32 & -90 & 18 \\
\hline & $\mathrm{R}$ & $17 / 18$ & $20.22^{* * *}$ & 4 & -92 & -6 \\
\hline & & 18 & $18.58^{* * *}$ & 44 & -78 & -8 \\
\hline \multirow[t]{2}{*}{ Fusiform gyrus } & L & $19 / 37$ & $19.53^{* * *}$ & -26 & -48 & -6 \\
\hline & & 20 & $18.77^{* * *}$ & -30 & -44 & -18 \\
\hline \multirow[t]{2}{*}{ Parahippocampal cortex } & $\mathbf{R}$ & 19 & $21.20 * * *$ & 28 & -60 & -8 \\
\hline & & 20 & $16.48^{* * *}$ & 36 & -40 & -18 \\
\hline \multirow[t]{4}{*}{ Hippocampus } & $\mathbf{L}$ & - & $12.86^{* * *}$ & -28 & -28 & -12 \\
\hline & & - & $13.25^{* * *}$ & -24 & -20 & -18 \\
\hline & $\mathbf{R}$ & - & $12.99 * * *$ & 26 & -24 & -16 \\
\hline & & - & $13.54 * * *$ & 24 & -30 & -6 \\
\hline \multirow[t]{2}{*}{ Ventral lateral PFC } & L & 45 & $11.22^{* * *}$ & -28 & 24 & \\
\hline & $\mathrm{R}$ & 47 & $10.16^{* * *}$ & 30 & 32 & -10 \\
\hline \multirow[t]{2}{*}{ Perirhinal cortex } & L & 21 & $8.81^{* * *}$ & -36 & -10 & -28 \\
\hline & $\mathrm{R}$ & 21 & $8.11^{* * *}$ & 38 & -8 & -28 \\
\hline \multirow[t]{2}{*}{ Midbrain/brainstem } & L & - & $8.01^{* * *}$ & -2 & -28 & -12 \\
\hline & $\mathrm{R}$ & - & $7.68 * * *$ & -2 & -34 & -32 \\
\hline
\end{tabular}

Local maxima are defined in MNI152 standard space as implemented in SPM5. ${ }^{* *} p<0.05$, cluster level of the whole brain familywise error corrected. Activations in the MTL are in bold type. L, Left; R, right; BA, Brodmann area; MNI, Montreal Neurological Institute.

$p<0.001)$. The distribution of trial-based detail memory accuracy scores is shown in Table 3.

Next, we investigated the relationship between the subjective confidence of remembering the gist and the objective detail memory accuracy (and proportion correct). As can be seen in Table 2, detail memory accuracy (and proportion correct) only weakly increased with levels of subjective confidence. To test this latter relationship more formally, we calculated the correlation between subjective confidence ratings and detail memory accuracy on a trial-by-trial basis for each participant. The averaged Fisher's $z$-transformed correlation was $0.19 \pm 0.02$ (mean \pm SEM; calculated across participants), which was statistically different from zero $\left(t_{(19)}=7.91, p<0.001\right)$. Thus, as expected, subjective and objective measures were correlated significantly, but also contained a substantial proportion of nonshared variance.

\section{Neuroimaging results from parametric models}

First, we contrasted all encoding trials with low-level baseline fixation. This contrast revealed a set of widely distributed regions, including occipitotemporal areas extending into the MTL, the bilateral PFC, and other regions (Table 4). Subsequently, by contrasting trials that were later remembered with those later forgotten based on recognition of the one-sentence gist descriptions, we found subsequent memory effects in the bilateral hippocampus and the left ventral lateral and ventral medial PFC (Table 5). These effects are in line with previous studies showing medial temporal and prefrontal involvement in memory formation.

More important for the question at issue, we investigated which brain regions exhibited activity that was positively correlated with subsequent ratings of subjective confidence for remembering the gist. This parametric analysis was restricted to trials of which the one-sentence gist description was successfully identified (or remembered), and was performed after partialing out objective measure (i.e., accuracy scores of detail memory) using linear orthogonalization. This analysis yielded positive correlations between levels of subjective measure and activity in the left ventral lateral PFC, and the left posterior superior temporal gyrus extending into the lateral parietal cortex (at the temporo-
Table 5. Brain activations related to memory formation in parametric analyses

\begin{tabular}{|c|c|c|c|c|c|c|}
\hline \multirow[b]{2}{*}{ Brain regions } & \multirow[b]{2}{*}{$\mathrm{R} / \mathrm{L}$} & \multirow[b]{2}{*}{ BA } & \multirow[b]{2}{*}{$t$ values } & \multicolumn{3}{|c|}{ MNI152 coordinates } \\
\hline & & & & $x$ & $y$ & $z$ \\
\hline \multicolumn{7}{|c|}{ Subsequent memory effect: remembered vs forgotten } \\
\hline Posterior fusiform gyrus & $\mathrm{R}$ & 19 & $5.66^{* * *}$ & 40 & -68 & -10 \\
\hline Parahippocampal cortex & $\mathbf{L}$ & $36 / 37$ & $4.61^{* *}$ & -38 & -34 & -8 \\
\hline \multirow[t]{3}{*}{ Hippocampus } & $\mathbf{L}$ & - & $4.31^{* *}$ & -30 & -26 & -10 \\
\hline & & - & $4.50^{* *}$ & -16 & -34 & 0 \\
\hline & $\mathbf{R}$ & - & $4.39 * *$ & 26 & -24 & -14 \\
\hline Ventral lateral PFC & $\mathrm{L}$ & 45 & $3.86^{*}$ & -40 & 34 & -6 \\
\hline Ventral medial PFC & $\mathrm{L}$ & 10 & $3.99^{*}$ & -8 & 46 & -8 \\
\hline \multicolumn{7}{|c|}{ Positive correlations with subjective measure (confidence of remembering the gist) } \\
\hline Temporoparietal junction & $\mathrm{L}$ & 39 & $6.57^{* * *}$ & -48 & -60 & 22 \\
\hline \multirow[t]{2}{*}{ Posterior ITC } & $\mathrm{R}$ & 19 & $5.75^{* * *}$ & 50 & -68 & -12 \\
\hline & & 37 & $5.27^{* * *}$ & 48 & -56 & -14 \\
\hline \multirow[t]{2}{*}{ Ventral lateral PFC } & $\mathrm{L}$ & 47 & $5.08^{* * *}$ & -46 & 22 & -2 \\
\hline & & 45 & $4.52^{* * *}$ & -52 & 24 & 10 \\
\hline \multicolumn{7}{|c|}{ Positive correlation with objective measure (the number of details accurately remembered) } \\
\hline \multirow[t]{2}{*}{ Parahippocampal cortex } & $\mathbf{L}$ & $20 / 37$ & $6.75^{* * *}$ & -30 & -36 & -14 \\
\hline & $\mathbf{R}$ & $20 / 36$ & $5.45^{* * *}$ & 36 & -36 & -14 \\
\hline Angula & $\mathrm{L}$ & 21 & $5.75^{* * *}$ & -28 & -76 & 34 \\
\hline Hippocampus & $\mathbf{L}$ & - & $4.08^{* *}$ & -22 & -14 & -14 \\
\hline Ventral lateral PFC & $\mathrm{L}$ & 45 & $4.40^{*}$ & -44 & 36 & -12 \\
\hline
\end{tabular}

Local maxima are defined in MNI152 standard space as implemented in SPM5. ${ }^{* * *} p<0.05$ at cluster level of the whole brain corrected; activations in the MTL are in bold; ${ }^{* *} p<0.05$, cluster level of small volume corrected; ${ }^{*} p<$ 0.001, uncorrected; ITC, inferior temporal cortex; L, left; R, right; BA, Brodmann area; MNI, Montreal Neurological Institute.

parietal junction) (Table 5, Fig. 2A). Thus, these results indicate that activation of these regions specifically predicted levels of subjective sense of memory strength.

Subsequently, we identified brain regions specifically associated with objective measure of the number of details accurately retrieved. This analysis was again limited to trials of which the one-sentence gist description was remembered, but now the accuracy scores of objective measure were orthogonalized with respect to scores of subjective confidence. Clusters of voxels exhibiting a positive correlation with objective measure were found in the bilateral parahippocampal cortex, extending into the hippocampus and the fusiform gyrus, and in the left anterior hippocampus (Fig. 2 B). Additionally, one significant cluster was found outside of the MTL in the left anterior middle temporal gyrus (Table 5).

Given a concern that the orthogonalization procedure may force a dissociation between neural processes related to subjective and objective measurements, we also performed separate eventrelated parametric analyses using raw scores of these two measurements (i.e., without partialing out their mutual correlations). The differences between these analyses with and without orthogonalization were negligible. Moreover, we ran the same analyses with proportion correct scores (calculated using the method of hit rate divided by hit rate plus false alarm rate) as parametric modulations. These analyses yielded almost identical results and are therefore not reported in detail.

\section{Neuroimaging results from categorical model}

We next extracted parameter estimates from our main regions of interest in the PFC and MTL to further characterize and illustrate the distinct patterns of these regions related to subjective confidence of remembering the gist and objective detail memory measures. Figure 3 shows averaged parameter estimates after trials were grouped into four categories based on higher versus lower scores on subjective and objective measures, respectively (i.e., high-high, high-low, low-high, or low-low). Further statistical analysis of these extracted data also revealed that the left ventral 
A Subjective confidence (gist)

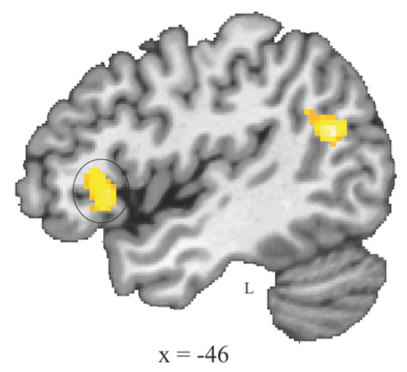

B Objective detail memory
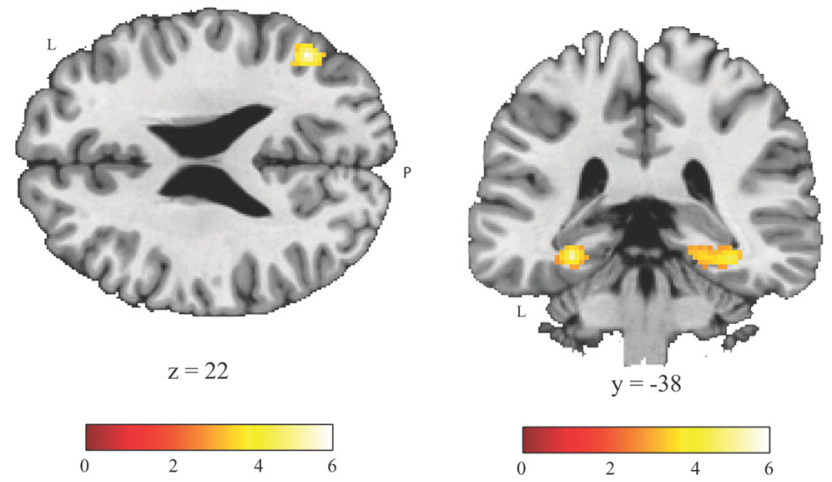

Figure 2. Brain activation related to subsequent subjective (confidence of remembering the gist) and objective (detail memory accuracy or the amount of information accurately remembered) measures in the parametric models. Statistical parametric maps are overlaid onto a high-resolution T1 template in MN1152 space (cluster-level $p<0.05$, corrected). $\boldsymbol{A}$, Areas exhibiting a positive correlation with trial-based subjective confidence of remembering the gist, including the left ventral lateral PFC (marked by a circle; left upper panel) and the left temporoparietal junction (left lower). $\boldsymbol{B}$, Areas exhibiting a positive (partial) correlation with trial-based objective detail memory measures including the left hippocampus (marked by a circle; right upper panel) and the bilateral parahippocampal cortex (right lower). L, Left; P, posterior.

lateral PFC exhibited a main effect of subjective confidence of remembering the gist $\left(F_{(1,19)}=6.72, p=0.018\right)$. However, neither the main effect of objective detail memory nor the interaction of both factors (both $F_{(1,19)}<1.90$, both $p>0.18$ ) reached significance. In contrast, both the hippocampus and the parahippocampal cortex showed a main effect of objective detail memory (both $F_{(1,19)}>16.60$, both $p<0.001$ ), but neither a significant main effect of subjective confidence of gist memory nor an interaction effect (all $F_{(1,19)}<1$ ). These results again indicate that ventral lateral PFC activity predicted subsequent subjective confidence of remembering the gist, whereas hippocampal and parahippocampal activity predicted levels of subsequent objective detail memory measures or the amount of information remembered (Fig. 3A,B).

\section{Discussion}

Here, we dissociated neural correlates of successful memory formation related to a subsequent subjective sense of memory strength from those related to an objective measure of the number of details accurately remembered. While activation of the left ventral lateral PFC and the temporoparietal junction predicted subsequent levels of subjective confidence when remembering the gist of a past event, parahippocampal and hippocampal activity predicted the number of details accurately remembered. Several recent functional neuroimaging studies using conventional recognition memory paradigms have addressed neural processes regulating memory strength, such as strong versus weak memories (Squire et al., 2007; Kirwan et al., 2008; Shrager et al., 2008)
A Objective detail memory Left ventral lateral PFC

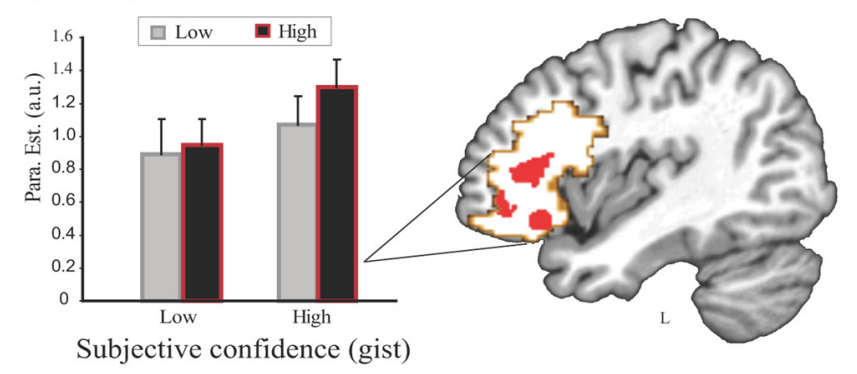

B

Objective detail memory

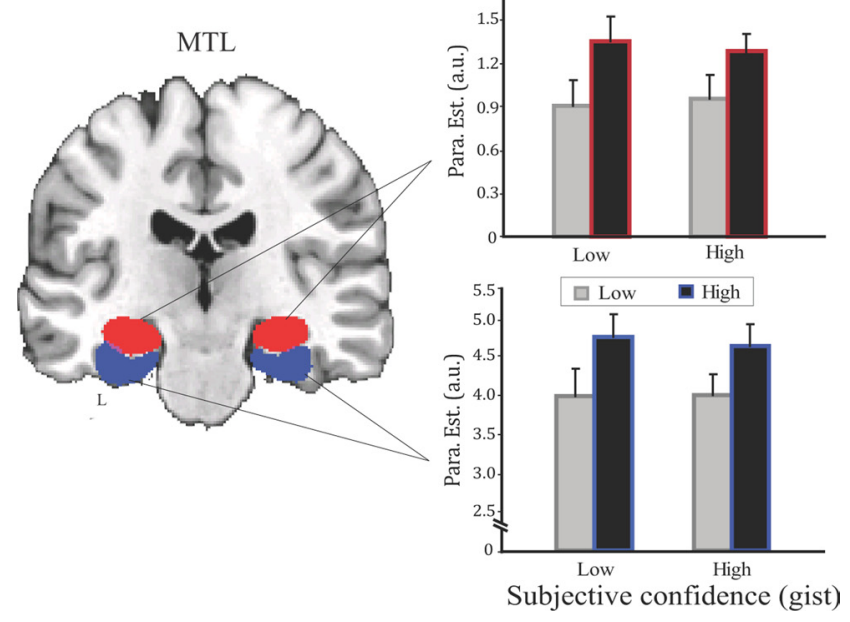

Figure 3. Data from the categorical model showing the left ventral lateral PFC and the MTL activity associated with subjective (confidence of remembering the gist) and objective (detail memory accuracy or the amount of information accurately remembered) measures. $A$, Left, The left ventral lateral prefrontal activity showed a main effect of subjective confidence but not objective detail memory measures. Right, Combination of anatomically defined (coded in light red) and functionally defined (based on the contrast of overall activation vs fixation baseline; coded in light gray) ventral lateral PFC overlaid onto a high-resolution T1 template in MNI152 space. $\boldsymbol{B}$, Left, Anatomically defined bilateral hippocampus (coded in red) and bilateral parahippocampal cortex (PHC) (coded in blue) overlaid onto a high-resolution T1 template in MNI152 space. Right, Hippocampal (top right) as well as parahippocampal (bottom right) activity predicted levels of subsequent objective detail memory accuracy regardless of subjective confidence of remembering the gist. High (Low) represents higher (lower) scores for subjective confidence and objective detail memory measures. Error bars represent corresponding SEM.

and memory retrieval associated with high versus low amount of information recollected (Vilberg and Rugg, 2007, 2009). Our findings importantly extend these studies by showing that such neural operations can be subdivided into component processes that are associated with distinct dimensions of memory strength.

Behaviorally, it has been long suggested that the subjective confidence of remembrance and the objective veridicality of a memory are not always correlated with each other. For instance, introspective recollection with high subjective confidence occurs in the absence of veridicality, as exemplified by eyewitness misidentification or false memories (Loftus, 1979; Schacter, 2001; Kensinger, 2008). Such experimental findings and real-life phenomena are consistent with notions that representations of a memory are compositional and nonunitary (Henke, 2010). One may therefore argue that the concept of memory strength reflects a functionally heterogeneous set of phenomena, which can be dissociated at least along the dimensions of the subjective sense of (gist) memory strength and the objective amount of information accurately remembered implemented in our present study. 
The distinction between subjective sense of memory strength and the objective amount of information remembered may reflect distinct components of a compositional memory (Schacter and Addis, 2007; Henke, 2010), such as the gist and corresponding specific details (or objective memory contents). This deviates from a widely investigated dissociation along the lines of familiarity and recollection (Yonelinas et al., 1999; Yonelinas, 2001; Eichenbaum et al., 2007). Notably, our design was not based on a conventional recognition memory paradigm, but on a paradigm that required explicit recall of both the gist of the studied scenes and their corresponding details based on written descriptions. Nevertheless, we cannot fully exclude the possibility that the gist memory test may contain some familiarity, because participants could have made their memory judgment based in part on the sense of familiarity of a mental image constructed from the gist descriptions. Regardless of this potential contamination, it is easily conceivable that both subjective and objective measures implemented here are dominated by recollective processes of imagery and scene reconstruction. Thus, our observed distinction between neural correlates of subjective sense of (gist) memory strength and objective amount of information accurately remembered does not readily map onto a framework of familiarity versus recollection.

As shown by our findings, activity in specific frontal and parietal regions contributes to memory strength by predicting the subjective sense of confidence associated with retrieval of the gist of an episode. These regions have been associated with several memory- and metamemory-related processes such as semantic processing, internal monitoring, and allocation of attentional resources (Wagner, 1999; Metcalfe, 2000; Miller and Cohen, 2001; Simons and Spiers, 2003; Cabeza et al., 2008). Such processes are thought to facilitate the rapid capture of the semantic gist of complex visual information, or the creation of a proactive sense for a novel experience (Bar, 2003, 2009). Notably, such higher semantic processes differ from the establishment of familiarity, in which the perirhinal cortex is implicated (Diana et al., 2007; Eichenbaum et al., 2007). By using a conventional recognition memory paradigm with confidence ratings, a study by Preston et al. (2010) demonstrates that parahippocampal and hippocampal activity predicted levels of confidence when recognizing scenes. We do not think, however, that this finding speaks against our finding that frontal and temporoparietal activity, but not medial temporal activity, predicts subjective sense of (gist) memory strength. The paradigm they used was designed to investigate whether there are domain-specific or domain-general mnemonic processes in parahippocampal and hippocampal regions, rather than disentangling subjective confidence ratings from the objective amount of information accurately encoded. Thus, it is well possible that high levels of confidence for correctly recognized scenes were driven by encoding of many specific details. In our present study, however, we developed a novel paradigm to qualify subjective sense of memory strength, in which participants were asked to identify one-sentence written gist descriptions of studied scenes in the gist memory test rather than recognizing original studied stimuli. In this type of memory test, remembering the gist of an episode is thought to be independent of specific details (Adolphs et al., 2001, 2005). Hence, high levels of confidence for remembering the gist would mainly reflect a strong subjective sense of memory strength without being confounded by retrieving specific details. In other words, although remembering the gist of a past event may lead to a strong subjective sense of remembrance, this is not necessarily correlated with verbatim details. For instance, patients with parietal lesions show reduced confidence in remembering their previous experiences, whereas their overall memory performance remains intact (Simons and Spiers, 2003; Simons et al., 2010). Therefore, a strong subjective sense of memory strength may be a consequence of frontal-parietal processes involved in top-down modulation of memory- and metamemory-related processes such as semantic processing, internal monitoring, and allocation of attentional resources that contribute to facilitating the reconstruction of visual scenes into general semantic categories.

The parahippocampal cortex and the hippocampus appear to form memories about details and their association with a specific event (here, a picture of a complex scene), which may be mediated by relational binding mechanisms in the MTL (Davachi, 2006; Eichenbaum et al., 2007; Squire et al., 2007). In our study, memory strength was objectively measured by assessing the number of details that were recalled accurately in a subsequent memory test. In terms of a recent neurocognitive model, memory strength can be objectively determined by the number of feature units that can be successfully reinstated during retrieval (Shimamura and Wickens, 2009). To form a strong, objectively defined memory, neural resources are required that bind disparate details (or feature units) into a coherent episodic memory. The more such resources are involved when forming a new episodic memory, the more feature units of that episode are established, and thus the more details can be remembered. The hierarchical organization of the parahippocampal regions and the hippocampus, in which neural processing streams from diverse association areas converge, provides an optimal substrate for such processes to occur (Squire et al., 2004; Suzuki and Amaral, 2004; Fernández and Tendolkar, 2006; Suzuki and Baxter, 2009).

The dissociation along the two distinct dimensions, i.e., subjective confidence based on introspective experience of the gist and the objective amount of specific details accurately remembered, may contribute to the flexibility of our memory processes. The frontoparietal contribution, predicting the subjective sense of memory strength, is likely beneficial for rapidly extracting the gist of a complex event to support a quick categorical decision or prediction when it is necessary (Bar, 2003, 2009). The MTL, in contrast, appears to contribute to building memories about details so that accurate and vivid retrieval of past events can be retrieved. It remains open, however, how these two (sets of) processes are coordinated when new memories are formed. In some instances, the optimal balance between them may be altered. One may speculate that arousal or acute stress might affect this balance and result in dissociation between subjective sense of remembrance and the objective memory content(s) remembered. A potent example of this is eyewitness misidentification, where a high subjective sense of confidence for correct recollection (i.e., the gist) occurs in the absence of objective veridicality of specific memory details (Loftus, 1979; Schacter, 2001; Sharot et al., 2007; Kensinger, 2008; Phelps and Sharot, 2008; Simons et al., 2010). It would be relevant for future research to address how the brain achieves an optimally adaptive balance between these distinct (sets of) processes when individuals are exposed to an acute stressor.

In sum, this study provides initial evidence for the notion that memory strength may reflect a functionally heterogeneous set of phenomena, and can be at least defined along the dimensions of subjective sense of remembrance and the objective memory content(s). The subjective sense of memory strength appears established by frontal-parietal processing and determines how strong a memory (i.e., the gist) is perceived at retrieval. The other and more objective dimension is associated with medial-temporal processing and determines how accurate specific details or infor- 
mation are later remembered within the context of a specific study episode. This is in line with recent theoretical proposals that memory representations (or traces) are compositional and nonunitary. Further research is needed to understand how the balance between these multiple dimensions is achieved during memory formation.

\section{References}

Adolphs R, Denburg NL, Tranel D (2001) The amygdala's role in long-term declarative memory for gist and detail. Behav Neurosci 115:983-992.

Adolphs R, Tranel D, Buchanan TW (2005) Amygdala damage impairs emotional memory for gist but not details of complex stimuli. Nat Neurosci 8:512-518.

Bar M (2003) A cortical mechanism for triggering top-down facilitation in visual object recognition. J Cogn Neurosci 15:600-609.

Bar M (2009) The proactive brain: memory for predictions. Philos Trans R Soc Lond B Biol Sci 364:1235-1243.

Brett M, Anton JL, Valabregue R, Poline JB (2002) Region of interest analysis using an SPM toolbox. Neuroimage 16:abstract 497.

Cabeza R, Rao SM, Wagner AD, Mayer AR, Schacter DL (2001) Can medial temporal lobe regions distinguish true from false? An event-related functional MRI study of veridical and illusory recognition memory. Proc Natl Acad Sci U S A 98:4805-4810.

Cabeza R, Ciaramelli E, Olson IR, Moscovitch M (2008) The parietal cortex and episodic memory: an attentional account. Nat Rev Neurosci 9:613-625.

Davachi L (2006) Item, context and relational episodic encoding in humans. Curr Opin Neurobiol 16:693-700.

Diana RA, Yonelinas AP, Ranganath C (2007) Imaging recollection and familiarity in the medial temporal lobe: a three-component model. Trends Cogn Sci 11:379-386.

Ebbinghaus H (1885) Memory: a contribution to experimental psychology. Reprint (Ruger HA, Bussenius CE, translators). New York: Teachers College, Columbia University, 1913.

Eichenbaum H, Yonelinas AP, Ranganath C (2007) The medial temporal lobe and recognition memory. Annu Rev Neurosci 30:123-152.

Fernández G, Tendolkar I (2006) The rhinal cortex: 'gatekeeper' of the declarative memory system. Trends Cogn Sci 10:358-362.

Friston KJ, Holmes AP, Worsley KJ, Poline JB, Frith C, Frackowiak RSJ (1995) Statistical parametric maps in functional imaging: a general linear approach. Hum Brain Mapp 2:189-210.

Henke K (2010) A model for memory systems based on processing modes rather than consciousness. Nat Rev Neurosci 11:523-532.

Kao YC, Davis ES, Gabrieli JD (2005) Neural correlates of actual and predicted memory formation. Nat Neurosci 8:1776-1783.

Kensinger EA (2008) Emotional memory across the adult life span. London: Psychology Press.

Kim H, Cabeza R (2007) Trusting our memories: dissociating the neural correlates of confidence in veridical versus illusory memories. J Neurosci 27:12190-12197.

Kirwan CB, Wixted JT, Squire LR (2008) Activity in the medial temporal lobe predicts memory strength, whereas activity in the prefrontal cortex predicts recollection. J Neurosci 28:10541-10548.

Loftus E (1979) Eyewitness reliability. Science 205:386-387.

Metcalfe J (2000) Metamemory: theory and data. In: The Oxford handbook of memory (Tulving E, Craik FIM, eds), pp 197-211. New York: Oxford UP.

Miller EK, Cohen JD (2001) An integrative theory of prefrontal cortex function. Annu Rev Neurosci 24:167-202.

Phelps EA, Sharot T (2008) How (and why) emotion enhances the subjective sense of recollection. Curr Dir Psychol Sci 17:147-152.

Qin S, Rijpkema M, Tendolkar I, Piekema C, Hermans EJ, Binder M, Pe- tersson KM, Luo J, Fernández G (2009) Dissecting medial temporal lobe contributions to item and associative memory formation. Neuroimage 46:874-881.

Schacter DL (1995) Memory distortion: how minds, brains, and societies reconstruct the past. Cambridge, MA: Harvard UP.

Schacter DL (2001) The seven sins of memory: how the mind forgets and remembers. Boston: Houghton Mifflin.

Schacter DL, Addis DR (2007) Constructive memory: the ghosts of past and future. Nature 445:27.

Schacter DL, Curran T, Galluccio L, Milberg WP, Bates JF (1996) False recognition and the right frontal lobe: a case study. Neuropsychologia 34:793-808.

Sharot T, Martorella EA, Delgado MR, Phelps EA (2007) How personal experience modulates the neural circuitry of memories of September 11. Proc Natl Acad Sci U S A 104:389-394.

Shimamura AP, Wickens TD (2009) Superadditive memory strength for item and source recognition: the role of hierarchical relational binding in the medial temporal lobe. Psychol Rev 116:1-19.

Shrager Y, Kirwan CB, Squire LR (2008) Activity in both hippocampus and perirhinal cortex predicts the memory strength of subsequently remembered information. Neuron 59:547-553.

Simons JS, Spiers HJ (2003) Prefrontal and medial temporal lobe interactions in long-term memory. Nat Rev Neurosci 4:637-648.

Simons JS, Peers PV, Mazuz YS, Berryhill ME, Olson IR (2010) Dissociation between memory accuracy and memory confidence following bilateral parietal lesions. Cereb Cortex 20:479-485.

Squire LR, Stark CE, Clark RE (2004) The medial temporal lobe. Annu Rev Neurosci 27:279-306.

Squire LR, Wixted JT, Clark RE (2007) Recognition memory and the medial temporal lobe: a new perspective. Nat Rev Neurosci 8:872-883.

Suzuki WA, Amaral DG (2004) Functional neuroanatomy of the medial temporal lobe memory system. Cortex 40:220-222.

Suzuki WA, Baxter MG (2009) Memory, perception, and the medial temporal lobe: a synthesis of opinions. Neuron 61:678-679.

Tulving E (1981) Similarity relations in recognition. J Verb Learn Verb Behav 20:479-496.

Tzourio-Mazoyer N, Landeau B, Papathanassiou D, Crivello F, Etard O, Delcroix N, Mazoyer B, Joliot M (2002) Automated anatomical labeling of activations in SPM using a macroscopic anatomical parcellation of the MNI MRI single-subject brain. Neuroimage 15:273-289.

Vilberg KL, Rugg MD (2007) Dissociation of the neural correlates of recognition memory according to familiarity, recollection, and amount of recollected information. Neuropsychologia 45:2216-2225.

Vilberg KL, Rugg MD (2009) Left parietal cortex is modulated by amount of recollected verbal information. Neuroreport 20:1295-1299.

Wagner AD (1999) Working memory contributions to human learning and remembering. Neuron 22:19-22.

Wixted JT, Ebbesen EB (1991) On the form of forgetting. Psychol Sci 2:409-415.

Wixted JT, Mickes L, Squire LR (2010) Measuring recollection and familiarity in the medial temporal lobe. Hippocampus 20:1195-1205.

Worsley KJ, Marrett S, Neelin P, Vandal AC, Friston KJ, Evans AC (1996) A unified statistical approach for determining significant signals in images of cerebral activation. Hum Brain Mapp 4:58-73.

Yonelinas AP (2001) Components of episodic memory: the contribution of recollection and familiarity. Philos Trans R Soc Lond B Biol Sci 356:1363-1374.

Yonelinas AP, Kroll NE, Dobbins IG, Soltani M (1999) Recognition memory for faces: when familiarity supports associative recognition judgments. Psychon Bull Rev 6:654-661. 